\section{Sustained moderate visual loss as a predictive end point for visual loss in non-proliferative diabetic retinopathy}

A Girach¹, LP Aiello², RC Milton ${ }^{3}$, MD Davis ${ }^{4}$, RP Danis ${ }^{4}, X$ Zhi $^{5}$, MJ Sheetz ${ }^{5}$ and L Vignati ${ }^{6}$ for the PKC-DRS2 Study Group

\begin{abstract}
Purpose In PKC-DRS2, the efficacy of the oral PKC- $\beta$ inhibitor, ruboxistaurin $32 \mathrm{mg} / \mathrm{day}$, was measured by the primary end point of sustained moderate visual loss (SMVL: $a \geqslant 15$ letter decrease from baseline on the ETDRS (Early Treatment Diabetic Retinopathy Study) chart sustained at least for the last 6 months of study participation). We now evaluate whether SMVL is more accurate than moderate visual loss (MVL: a single occurrence of a decrease from baseline of $\geqslant 15$ ETDRS letters) for predicting future visual loss.

Methods Study eyes with moderately severe to very-severe non-proliferative diabetic retinopathy, best-corrected visual acuity of at least 45 letters on the ETDRS chart ( $\sim$ Snellen 20/125), and no prior pan retinal photocoagulation were evaluated in 506 patients (869 eyes) who completed 36 months of treatment.

Results Sixty-five percentage (26/40) of study eyes with the onset of SMVL within 24 months of enrolment still had SMVL at study completion (36 months). In comparison, only $24 \%$ (30/126) with MVL within 24 months had SMVL at study completion. Analyses based on data from 6, 12, and 18 months of treatment were similar. Conclusions SMVL is a more predictable measure of subsequent visual loss than is a single time point measure of MVL.

Eye (2009) 23, 209-214; doi:10.1038/eye.2008.324; published online 7 November 2008
\end{abstract}

Keywords: diabetic retinopathy; visual loss; diabetes

\section{Introduction}

Diabetic retinopathy (DR) affects nearly $40 \%$ of patients with diabetes. ${ }^{1}$ Patients with non-proliferative DR (NPDR) are at increased risk for visual loss, which can lead to severe morbidity and loss of independence. ${ }^{2}$ The current options for treating and preventing DR include blood glucose, blood pressure, and lipid control, pan retinal photocoagulation (PRP) for high-risk proliferative DR (PDR), focal or grid photocoagulation for eyes with clinically significant macular oedema (CSME), and vitrectomy for severe PDR or vitreous haemorrhage. Although the effectiveness of these treatments has been documented in randomized, controlled clinical trials, ${ }^{3-9} \mathrm{DR}$ remains the leading cause of blindness in the working-age population of the United States and developed nations. ${ }^{10,11}$

Medical treatments aimed at the prevention of retinopathy progression in the early stages of the disease are being studied in an attempt to further reduce the risk of visual loss. ${ }^{12}$ Given limited early symptoms, the substantial risks associated with advanced retinopathy, and the development of new pharmacotherapeutic strategies to treat DR, rigorous, early end points, predictive of future visual loss, would be of tremendous value in clinical evaluation of new therapies.

In the PKC-DRS2 trial, the efficacy of ruboxistaurin (RBX) $32 \mathrm{mg} /$ day, an oral PKC- $\beta$ inhibitor, compared with placebo was measured by using an end point based on sustained moderate visual loss (SMVL) for the last 6 months of study participation (ie, 30-36 months). SMVL was defined as a loss of $\geqslant 15$ letters on the ETDRS (Early Treatment Diabetic Retinopathy Study) chart sustained for 6 months. We now perform additional analyses to evaluate the durability of SMVL at multiple early time points as a measure to predict the visual loss after 3 years. We also compare the
${ }^{1}$ Department of Ophthalmology, Merck Research Laboratories, Hertfordshire, UK

${ }^{2}$ Department of Ophthalmology, Joslin Diabetes Center, Harvard Medical School, Boston, MA, USA

${ }^{3}$ EMMES Corporation, Rockville, MD, USA

${ }^{4}$ Department of Ophthalmology and Visual Sciences, University of Wisconsin School of Medicine and Public Health, Madison, WI, USA

${ }^{5}$ Eli Lilly and Company, Lilly Research Laboratories, Indianapolis, IN, USA

${ }^{6}$ Marcadia Biotech Inc., Carmel, IN, USA

Correspondence: A Girach, Merck Research Laboratories,

Hertford Road,

Hoddesdon,

Hertfordshire EN11 9BU, UK Tel: + 441992 467272;

E-mail: aniz_girach@ merck.com

Received: 16 April 2008 Accepted in revised form: 26 September 2008 Published online: 7 November 2008

This study was presented at the American Academy of Ophthalmology and Asian Pacific Academy of Ophthalmology Meeting; Las Vegas, NV, USA, on 11-14 November 2006 
relative predictive value of SMVL and MVL in this regard, and determine how various severities of visual loss present during the study were predictive of visual loss after 3 years.

\section{Materials and methods}

The study design of the PKC-DRS2 trial has been previously published in detail. ${ }^{13}$ Briefly, the PKC-DRS2 trial was a 36-month, multicenter, randomized, doublemasked, placebo-controlled phase 3 study. Patients were randomized to placebo $(n=340)$ or once daily RBX $32 \mathrm{mg}$ $(n=345)$. Major inclusion criteria were moderately severe to very-severe NPDR (ETDRS retinopathy score of $\geqslant 47 \mathrm{~A}$ and $\leqslant 53 \mathrm{E}$ ), no prior PRP in a study eye, and the bestcorrected visual acuity of at least 45 ETDRS letters (approximately 20/125 on the Snellen eye chart). The primary end point of the study was SMVL for the last 6 months of study participation (30-36 months) in at least one study eye in an intent-to-treat population. The PKCDRS2 trial strictly adhered to the principles of the Declaration of Helsinki statement and the guidelines on good clinical practice, and was approved by the Institutional Review Boards of all study sites. Written informed consent was obtained from all patients. We certify that all applicable institutional and governmental regulations concerning the ethical use of human volunteers were followed in this research.

In the current post hoc analyses, pooled results (placebo and RBX combined data) were examined to determine whether SMVL (during the study) or MVL (during the study) that began within $6,12,18$, and 24 months of study participation was a more rigorous end point for the prediction of visual loss after 3 years of study participation (SMVL at the end of the study). Only study eyes from the completer cohort of patients were analysed. An eye was included in the completer cohort, if the patients completed 3 years (36 months) of the treatment phase with either RBX or placebo. Data were analysed in terms of the proportion of eyes instead of patients. Treatment effect was not analysed, but data from patients receiving placebo were analysed separately to demonstrate whether or not treatment might have had an effect on the pooled results.

Analyses were cumulative and eyes were categorized into temporal categories (ie, within 6, 12, 18, or 24 months of baseline) based on the onset time of the visual loss during the study. For example, an eye with a single time point MVL that occurred by month 6 was considered to have 'MVL' and was counted in each of the temporal categories. An eye with sustained visual loss (for either 3 or 6 months) that began before or at month 6 was considered to have sustained visual loss in the 'within 6 months' and all subsequent temporal categories. An eye with loss that began at month 24 was counted only in the within 24-month temporal category.

The analyses based on the degree of visual loss (ie, $\geqslant 5$, $\geqslant 10$, or $\geqslant 15$ letter loss) were inclusive of categories of more visual loss. For example, an eye that had a loss of $\geqslant 15$ letters would also be counted in the $\geqslant 5$ and $\geqslant 10$ letter loss categories.

\section{Results}

In all, 685 patients were randomized into the PKC-DRS2 trial. Of these 685 patients, 506 patients (869 study eyes, 430 placebo, and $439 \mathrm{RBX} 32 \mathrm{mg}$ ) completed the 36 -month treatment phase and were included in the completer cohort.

\section{SMVL vs $M V L$}

Sixty-five percent (26/40) of the study eyes that had SMVL that began within 24 months of study participation also had SMVL at the 3-year study completion (Figure 1, Table 1). In comparison, only $24 \%$ (30/126) of study eyes with MVL (during the study) that began within 24 months had SMVL at the end of the

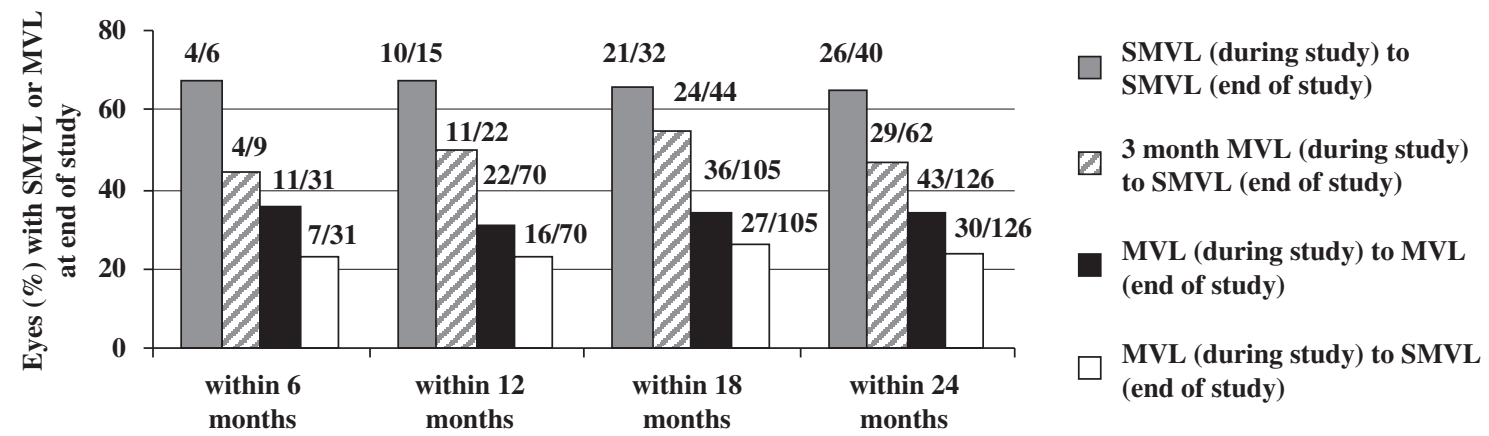

Figure 1 Proportion of eyes with SMVL (during the study) or MVL (during the study). Proportion of eyes as predictive end points at the end of the study, by the time period within which the visual loss first occurred. 
Table 1 Proportion of study eyes developing SMVL after 3 years of study participation by categories of visual loss experienced during the study

\begin{tabular}{|c|c|c|c|c|}
\hline \multirow{2}{*}{$\begin{array}{l}\text { Visual loss category } \\
\text { (during the study) }\end{array}$} & \multicolumn{4}{|c|}{ Time category ${ }^{\mathrm{a}}$} \\
\hline & Within 6 months & Within 12 months & Within 18 months & Within 24 months \\
\hline \multirow[t]{2}{*}{ MVL } & $23 \%(7 / 31)$ & $23 \%(16 / 70)$ & $26 \%(27 / 105)$ & $24 \%(30 / 126)$ \\
\hline & CI: $8-37 \%$ & CI: $13-33 \%$ & CI: $17-34 \%$ & CI: $16-31 \%$ \\
\hline \multicolumn{5}{|l|}{ 6-month sustained visual loss ${ }^{\dagger}$} \\
\hline \multirow[t]{2}{*}{ 15-letter loss (ie, SMVL) } & $67 \%(4 / 6)$ & $67 \%(10 / 15)$ & $66 \%(21 / 32)$ & $65 \%(26 / 40)$ \\
\hline & CI: $29-104 \%$ & CI: 43-91\% & CI: $49-82 \%$ & CI: $50-80 \%$ \\
\hline \multirow[t]{2}{*}{ 10-letter loss } & $41 \%(9 / 22)$ & $44 \%(21 / 48)$ & $40 \%(27 / 68)$ & $32 \%(30 / 94)$ \\
\hline & CI: $20-61 \%$ & CI: $30-58 \%$ & CI: $28-51 \%$ & CI: $22-41 \%$ \\
\hline \multirow{2}{*}{ 5-letter loss } & $24 \%(20 / 83)$ & $20 \%(26 / 130)$ & $17 \%(29 / 170)$ & $16 \%(32 / 207)$ \\
\hline & CI: $15-33 \%$ & CI: $13-27 \%$ & CI: $11-23 \%$ & CI: $11-20 \%$ \\
\hline \multicolumn{5}{|l|}{ 3-month sustained visual loss ${ }^{\dagger}$} \\
\hline \multirow[t]{2}{*}{ 15-letter loss (ie, 3-month MVL) } & $44 \%(4 / 9)$ & $50 \%(11 / 22)$ & $55 \%(24 / 44)$ & $47 \%(29 / 62)$ \\
\hline & CI: $12-77 \%$ & CI: $29-71 \%$ & CI: $40-69 \%$ & CI: $34-59 \%$ \\
\hline \multirow{2}{*}{ 10-letter loss } & $35 \%(10 / 29)$ & $34 \%(22 / 65)$ & $29 \%(27 / 93)$ & $24 \%(30 / 124)$ \\
\hline & CI: $17-52 \%$ & CI: $22-45 \%$ & CI: $20-38 \%$ & CI: $17-32 \%$ \\
\hline \multirow[t]{2}{*}{ 5-letter loss } & $18 \%(20 / 113)$ & $16 \%(27 / 172)$ & $13 \%(29 / 223)$ & $12 \%(32 / 267)$ \\
\hline & CI: $11-25 \%$ & CI: $10-21 \%$ & CI: $9-17 \%$ & CI: $8-16 \%$ \\
\hline
\end{tabular}

Abbreviations: $\mathrm{CI}=$ confidence interval (lower and higher bound of $95 \% \mathrm{CI}$ ); $\mathrm{MVL}=$ moderate visual loss; $\mathrm{SMVL}=$ sustained moderate visual loss (for 6 months of study participation).

${ }^{\text {a} E y e s ~ w e r e ~ c a t e g o r i z e d ~ i n t o ~ t i m e ~ c a t e g o r i e s ~ b a s e d ~ o n ~ t h e ~ o n s e t ~ d a t e ~ o f ~ t h e ~ M V L ~ o r ~ S M V L ~ d u r i n g ~ t h e ~ s t u d y . ~ A n a l y s e s ~ w e r e ~ c u m u l a t i v e ~ a n d ~ a n ~ e y e ~ c o u l d ~}$ have been counted in more than one category, for example, eyes in which a loss occurred during the first 6 months of follow-up were included in the 6-month and all subsequent time categories.

study. Analyses based on the data from eyes with an onset beginning within 6,12 , and 18 months postbaseline were similar.

The Kendall Tau correlation between SMVL during study participation and SMVL at study completion increased over the duration of study participation. The correlations between SMVL (during the study) that began within 12, 18, and 24 months of study participation and SMVL at the 3-year study completion were 0.34, 0.54, and 0.79 , respectively. These correlations were stronger than the correlations for MVL (during the study). The corresponding correlations between MVL (during the study) that began within 12,18, and 24 months of study participation and SMVL (at the end of the study) were $0.28,0.41$, and 0.41 , respectively.

SMVL (during the study) was also more predictive of SMVL (at the end of the study) when compared with MVL (during the study) as a predictive measure of MVL (at end of study). For example, only 34\% (36/105) of eyes that had MVL (during the study) by 18 months had MVL (at the end of the study). In comparison, 66\% (21/32) of eyes with the onset of SMVL (during the study) by 18 months of the study had SMVL (at the end of the study, Figure 1).

The duration of time that MVL (during the study) needs to be sustained to predict future visual loss was further analysed by considering an SMVL of 15 letters or more for $\geqslant 3$ months of study participation (ie, 3-month MVL). As shown in Figure 1 and Table 1, a 3-month MVL (during the study) was not as predictive as SMVL (during the study), although the 3-month MVL was more predictive than a single time point MVL during the study. The proportions of eyes with SMVL, 3-month MVL, or single time point MVL during the study that began within 24 months of study participation that had SMVL (at the end of the study) were $65 \%$ (26/40), 47\% (29/62), and 24\% (30/126), respectively. This trend remained consistent for analyses that considered 6, 12, and 18 months of study participation.

\section{Severity of visual loss}

As shown in Figure 2a and Table 1, a linear trend was seen when the relationship between the severity of sustained visual loss during study participation and SMVL (at the end of the study) was explored. For example, SMVL (at the end of the study) was most strongly predicted by a 6-month sustained visual loss of 15 letters or more (SMVL during the study) compared to a 6 -month sustained visual loss of $\geqslant 10$ or $\geqslant 5$ letters. The linear trend was consistent with the sustained visual loss within 6, 18, and 24 months of treatment. In addition, the same trend was observed for a 3-month sustained 

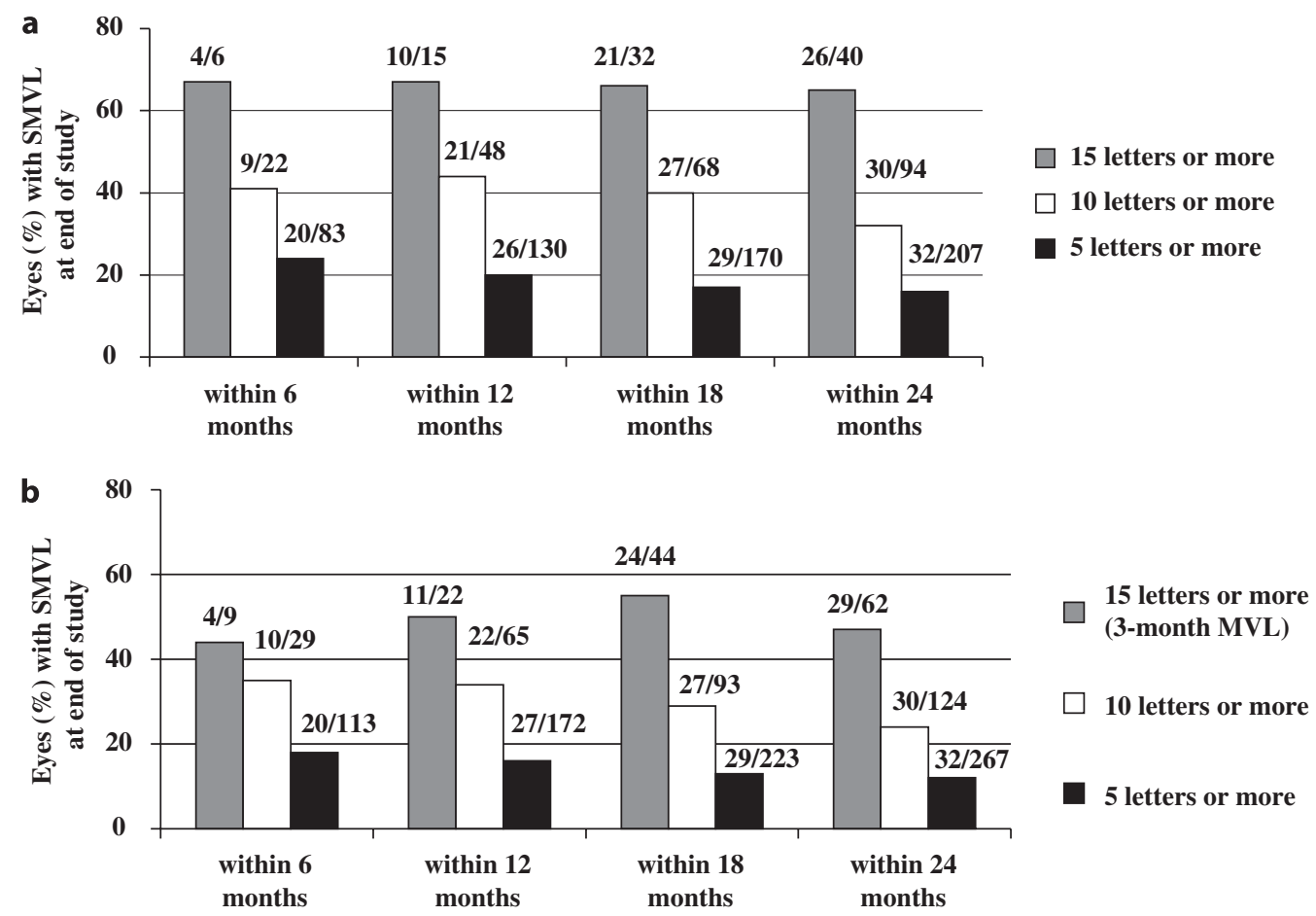

Figure 2 Proportion of eyes that had sustained $\geqslant 15, \geqslant 10$, and $\geqslant 5$ ETDRS letter loss and had SMVL at the end of the study. Proportion of eyes with (a) 6-month and (b) 3-month sustained ETDRS letter loss. Both figures refer to the time period within which the visual loss first occurred.

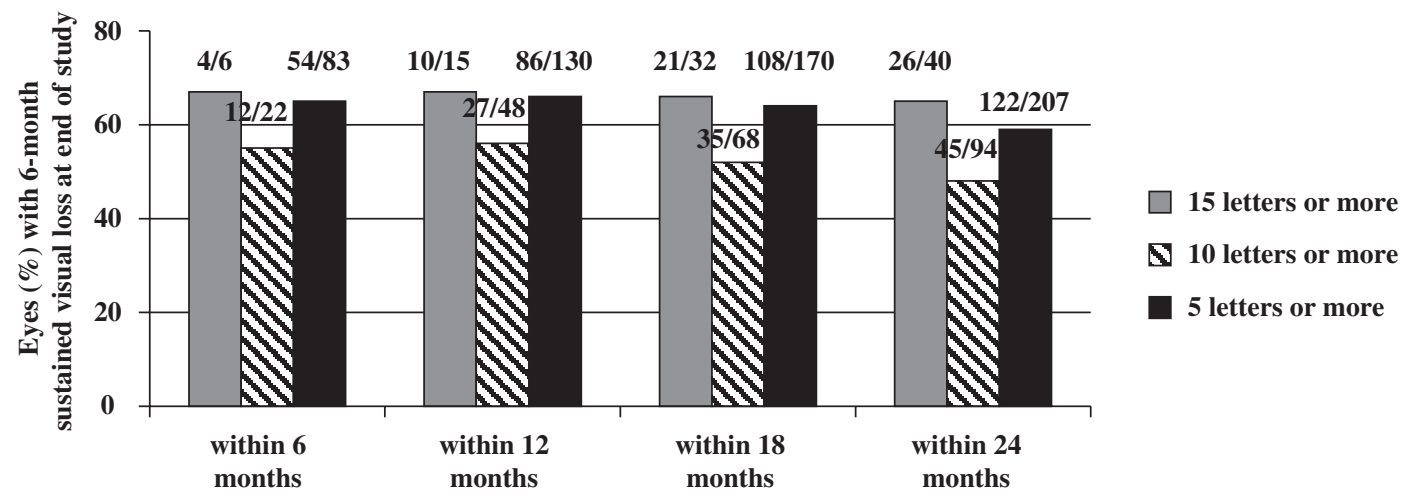

Figure 3 Proportion of eyes that had at least a 6 -month sustained $\geqslant 15, \geqslant 10$, and $\geqslant 5$ ETDRS letter loss. These eyes had the same severity of visual loss sustained for 6 months at the end of the study, by the time period within which visual loss first occurred.

visual loss, although 3 months of visual loss was less predictive of future visual loss than 6 months (see Figure 2a vs Figure 2b).

The ability of the 6-month sustained visual loss of $\geqslant 5, \geqslant 10$, or $\geqslant 15$ letters during study participation to predict the same visual loss outcome at the end of the 3 -year study was also studied (Figure 3). More than $50 \%$ of study eyes with sustained visual loss within 6 , 12,18 , and 24 months of study participation had sustained visual loss of the same severity at the end of the 3-year study.

\section{Placebo patients}

To investigate the possibility of RBX treatment effects on the pooled data analyses, analyses were completed in the subset of 430 study eyes of placebo-treated patients (Table 2). The same trends seen in the pooled data set were also seen in the analyses based on the placebotreated eyes only (see Table 1 vs Table 2). On the basis of the study eyes of placebo-treated patients, the ability of SMVL (during the study) to predict SMVL (at the end of the study) was also more then 2 times higher than 
Table 2 Proportion of study eyes of placebo-treated patients developing SMVL by categories of visual loss experienced during study participation

\begin{tabular}{|c|c|c|c|c|}
\hline \multirow{2}{*}{$\begin{array}{l}\text { Visual loss category } \\
\text { (during the study) }\end{array}$} & \multicolumn{4}{|c|}{ Time category ${ }^{\mathrm{a}}$} \\
\hline & Within 6 months & Within 12 months & Within 18 months & Within 24 months \\
\hline MVL & $35 \%(6 / 17)$ & $29 \%(10 / 35)$ & $33 \%(18 / 54)$ & $31 \%(20 / 65)$ \\
\hline \multicolumn{5}{|l|}{ 6-month sustained visual loss ${ }^{b}$} \\
\hline 15-letter loss (ie, SMVL) & $60 \%(3 / 5)$ & $60 \%(6 / 10)$ & $68 \%(13 / 19)$ & $64 \%(16 / 25)$ \\
\hline 10-letter loss & $47 \%(7 / 15)$ & $46 \%(12 / 26)$ & $43 \%(17 / 40)$ & $36 \%(20 / 56)$ \\
\hline 5-letter loss & $32 \%(12 / 37)$ & $27 \%(17 / 64)$ & $22 \%(19 / 87)$ & $21 \%(22 / 105)$ \\
\hline \multicolumn{5}{|l|}{ 3-month visual loss ${ }^{\mathrm{b}}$} \\
\hline 15-letter loss (ie, 3-month MVL) & $43 \%(3 / 7)$ & $50 \%(7 / 14)$ & $57 \%(16 / 28)$ & $51 \%(19 / 37)$ \\
\hline 10-letter loss & $42 \%(8 / 19)$ & $38 \%(13 / 34)$ & $33 \%(17 / 51)$ & $29 \%(20 / 68)$ \\
\hline 5-letter loss & $22 \%(12 / 54)$ & $20 \%(18 / 90)$ & $16 \%(19 / 119)$ & $16 \%(22 / 142)$ \\
\hline
\end{tabular}

Abbreviations: $\mathrm{MVL}=$ moderate visual loss; $\mathrm{SMVL}=$ sustained moderate visual loss for 6 months of study participation.

aEyes were categorized into time categories based on the onset date of the MVL or SMVL. Analyses were cumulative and an eye could have been counted in more than one category.

${ }^{\mathrm{b}}$ Analyses are cumulative. An eye could have been counted in more than one category.

MVL (during the study). Sixty-four percent (16/25) of study eyes of placebo-treated patients with SMVL (during the study) that began within 24 months of study participation had SMVL (at the end of the study) compared with $31 \%(20 / 65)$ of study eyes with MVL (during the study) within 24 months. In addition, SMVL (during the study) was more predictive of future visual loss than 3-month MVL in eyes of placebo-treated patients.

\section{Discussion}

MVL, as a standard measure of visual loss, was established by the ETDRS group and was defined as a doubling of the visual angle. ${ }^{8}$ This equates to an approximate loss of 15 letters on the ETDRS visual acuity chart. This is considered a clinically significant change of vision, eg, if a patient starts at $20 / 20$ (6/6 or 1.0) vision and his/her vision deteriorates by 15 letters (or 3-lines), then the patient's visual acuity will end up at 20/40 $(6 / 12$ or 0.5$)$. At this vision stage, the patient will not be able to legally drive without restrictions in most countries. ${ }^{14}$

In the randomized studies involving DR patients, the commonly accepted standard for a visual outcome end point is MVL. However, it is known that vision in patients that attain a 15-letter loss on the ETDRS chart can fluctuate over time for various metabolic and nonmetabolic reasons. Even a small fluctuation can easily result in inclusion or exclusion for a specific definite categorical cutoff point, such as a 15-letter loss mark. These changes expose the visual standard of MVL to visit-by-visit variability.

In designing the PKC-DRS2 trial, the end point of SMVL (at the end of the study) was chosen in an attempt to have a more rigorous and yet clinically meaningful end point. The end point of SMVL required MVL to be sustained for 6 months at the end of the 3-year study, similar to the concept of sustained DR progression utilized in the DCCT trial. ${ }^{15}$ Given that MVL may be transient, the end point of SMVL was hypothesized to be a more predictive long-term end point of visual loss. MVL sustained for 6 months at any time during the study (SMVL during the study) is a better predictor of sustained 6-month MVL at 3 years (SMVL at the end of the study) than isolated occurrences of MVL alone (Figure 1). SMVL, occurring as early as 12 months, is predictive of SMVL at 3 years (Figure 1). MVL sustained for 3 months compared to 6 months is a less robust predictor of SMVL at 3 years (Figure 1).

A 6-month sustained 15-letter loss (ie, SMVL) is a stronger risk factor than a 6-month sustained 10-letter loss, which in turn is stronger than a 6-month sustained 5-letter loss in predicting eventual visual loss at 3 years (Figure 2a). The corresponding 3-month sustained 15, 10, and 5-letter losses are not as predictive of eventual visual loss as the 6-month sustained equivalents (Figure 2b). It is also interesting to note that a 6-month sustained 5letter loss has approximately the same predictive value as a single time point MVL for eventual SMVL at 3 years. This is particularly evident for the earlier time points studied (Table 1).

In conclusion, clinical trials in DR may be reasonably designed to employ an end point of SMVL at an earlier time point than the current standard MVL at 3 years. SMVL (ie, MVL sustained for 6 months) appears to reduce the variability inherent in the measure of MVL alone and provides for a more reliable prediction of future sustained MVL. Thus, SMVL may be considered 
an appropriate end point for certain clinical trials involving visual acuity in patients with DR.

\section{Acknowledgements}

We acknowledge the support of April Boney of AJB Medical Writing Services and Keri Poi of Eli Lilly and Company. Matthew Sheetz is an employee of Eli Lilly and Company. Xin Zhi, Louis Vignati, Aniz Girach are former employees of Eli Lilly and Company. Lloyd Paul Aiello, Roy C Milton, Matthew D Davis, and Ronald P Danis are paid consultants for Eli Lilly and Company. This study was funded by Eli Lilly and Company.

\section{References}

1 Kempen JH, O'Colmain BJ, Leske MC, Haffner SM, Klein R, Moss SE et al. Eye Diseases Prevalence Research Group. The prevalence of diabetic retinopathy among adults in the United States. Arch Ophthalmol 2004; 122: 552-563.

2 Centers for Disease Control and Prevention (CDC). Blindness caused by diabetes-Massachusetts, 1987-1994. MMWR Morb Mortal Wkly Rep 1996; 45: 937-941.

3 Diabetes Control and Complications Trial Research Group: The effect of intensive treatment of diabetes on the development and progression of long-term complications in insulin-dependent diabetes mellitus. N Engl J Med 1993; 329: 977-986.

4 UK Prospective Diabetes Study (UKPDS) Group. Intensive blood-glucose control with sulphonylureas or insulin compared with conventional treatment and risk of complications in patients with type 2 diabetes (UKPDS 33). Lancet 1998; 352: 837-853.

5 UK Prospective Diabetes Study (UKPDS) Group. Tight blood pressure control and risk of macrovascular and microvascular complication in type 2 diabetes: UKPDS 38. BMJ 1998; 317: 703-713.

6 Diabetic Retinopathy Study Research Group. Preliminary report on effects of photocoagulation therapy. Am J Ophthalmol 1976; 81: 383-396.

7 Early Treatment Diabetic Retinopathy Study Research Group. Early photocoagulation for diabetic retinopathy: ETDRS report number 9. Ophthalmology 1991; 98(Suppl): 766-785.

8 Early Treatment Diabetic Retinopathy Study Research Group. Photocoagulation for diabetic macular edema: Early Treatment Diabetic Retinopathy Study report number 1. Arch Ophthalmol 1985; 103: 1796-1806.

9 The Diabetic Retinopathy Vitrectomy Study Research Group. Early vitrectomy for severe vitreous hemorrhage in diabetic retinopathy: two-year results of a randomized trial: Diabetic Retinopathy Vitrectomy Study report 2. Arch Ophthalmol 1985; 103: 1644-1652.

10 Klein R, Klein BE, Moss SE. The Wisconsin epidemiologic study of diabetic retinopathy: A review. Diab/Metab Rev 1989; 5: 559-570.

11 De La Cruz JP, Gonzalez-Correa JA, Guerrero A, De La Cuesta FS. Pharmacological approach to diabetic retinopathy. Diabetes Metab Res Rev 2004; 20: 91-113.

12 Fong DS. Changing times for the management of diabetic retinopathy. Surv Ophthalmol 2002; 47(Suppl 2): S238-S245.

13 The PKC-DRS2 Study Group. Effect of ruboxistaurin on visual loss in patients with diabetic retinopathy. Ophthalmology 2006; 113: 2221-2230.

14 International Council of Ophthalmology (ICO). Vision requirements for driver safety with emphasis on individual assessment (February, 2006). Available at: www.icoph.org/ standards.

15 Diabetes Control and Complications Trial Research Group. The effect of intensive diabetes treatment on the progression of diabetic retinopathy in insulin-dependent diabetes mellitus. Arch Ophthalmol 1995; 113: 36-51. 